\title{
EVOLUTION OF THE PROPERTIES OF NANOMATERIALS OBTAINED BY PULSED LASER ABLATION
}

\author{
S. FAIZULLAYEVA, S. M. KLIMENTOV, A. A. POPOV \\ National Research Nuclear University MEPhl, Moscow, Russian Federation, \\ Sabina.faizullaeva@gmail.com
}

https://doi.org/10.37904/nanocon.2019.8785

\begin{abstract}
The synthesis and application of metal and ceramic nanoparticle are significant subject in science and engineering. The metal nanoparticles such as gold, silver, and copper nanoparticles have more application in material science, nanomedicine, electronic, photonic, and art. One of the green method for preparation of metal nanoparticles is laser ablation technique that offers a unique tool for nanofabrication of nanoparticles. In this work, gold colloids were prepared by pulsed laser ablation of high purity gold plate immersed in deionized water and $10 \mu \mathrm{M} \mathrm{NaCl}$ solution. After ablation process we study the change in the size distribution of nanoparticles after the ablation process for a month.
\end{abstract}

Keywords: Laser ablation, gold nanoparticles, size distribution, colloidal solution, nanomedicine

\section{INTRODUCTION}

Colloidal solutions of plasmonic nanoparticles, such as gold and titanium nitride, have a number of unique properties, in particular, resonant absorption and scattering of light [1], as well as a huge amplification of the electromagnetic field near the surface [2], which makes them very effective both in catalysis [3] and in biomedical applications [4]. However, arbitrary transformations of the size, morphology and structure of nanoparticles during synthesis, and most importantly after its completion, during storage, temperature fluctuations and dilution of solutions is a significant problem. Uncontrolled and often unpredictable transformations lead to a loss of useful properties and a decrease in the reproducibility of the properties of nanoparticles, and hinder their practical application in biomedicine. Traditionally, aqueous solutions of nanoparticles are synthesized using well-studied methods of colloid chemistry [5]. However, the surface of nanomaterials thus obtained is often contaminated with residues of reagents, and the colloidal stability of solutions is achieved by coating their surfaces with stabilizing ligands, which reduce the catalytic activity of particles and impede their use in biological systems [6] (the reagents and ligands used are often biologically incompatible). In contrast to the chemical method, the physical method for the synthesis of colloidal solutions of nanoparticles, which appeared in 1993 and is based on laser ablation of a solid target immersed in a liquid, allows nanoparticles to be obtained under ultra-pure conditions, for example, in deionized water [7]. The colloidal stability of such solutions is achieved not due to the use of ligands, but because of the partial oxidation of the surface of nanoparticles [8]. As a result of oxidation, the surface receives an electric charge and the particles are electrostatically repelled from each other in the solution.

Often, the size and morphology of particles obtained by different authors under similar experimental conditions are significantly different from each other. The cause of such scatter can also be the "slow" maturation of nanoparticles, since the description of experiments almost always lacks an exact indication of the time interval from synthesis to characterization. Therefore, the study of the kinetics of "slow" maturation of colloidal solutions of nanoparticles based on titanium, gold, silicon, and a silicon-gold composite with the determination of the main physical mechanisms of transformation is an urgent and new study. A clear understanding of the physical mechanisms of "slow" particle growth, synthesized by laser methods, and the evolution of the constituents of the colloidal system will help find ways to increase its stability and improve the reproducibility of consumer properties of nanoparticle-based drugs for biomedical applications. 
The synthesis and application of metal and ceramic nanoparticles are significant subject in science and engineering. The metal nanoparticles such as gold, silver, and copper nanoparticles have more application in material science, nanomedicine, electronic, photonic, and art. One of the green method for preparation of metal nanoparticles is laser ablation technique that offers a unique tool for nanofabrication of nanoparticles.

It should be noted that in laser ablation mechanism selection of parameters of laser radiation (wavelength, pulse duration, its power) allows you to finely control the process of formation of nanostructures, and therefore, allows you to obtain materials with the required characteristics.

Laser ablation of solids in liquids is an effective technique with considerable potential in the generation of nanocrystals, which allows multilateral design through choosing appropriate solid target materials and confining liquids. In this work, gold colloids were prepared by pulsed laser ablation of high purity gold plate immersed in deionized water and $10 \mu \mathrm{M} \mathrm{NaCl}$ solution. The aim of this work is to study the time dependences of the size and morphological properties of simple gold-based nanoparticles obtained by femtosecond laser ablation.

\section{EXPERIMENTAL PART}

The use of pulsed-laser ablation at the solid-liquid interface was first reported by Patil and coworkers in 1987 to produce a metastable form of iron oxide from a pure iron target material [9]. Laser ablation in liquids has been used to produce nanoparticles as an alternative to chemicals because ablation in liquid is considered a cleaner environment in which to produce nanoparticles. Different liquids have different effects on the production of nanoparticles. For example, laser ablation of a Tin (Sn) target in water produces polycrystalline tin dioxide (SnO2) nanoparticles, while ablation in ethanol produces single crystals of tin coated with tin hydroxide $(\mathrm{Sn}(\mathrm{OH}) 2)$ nanoparticles [10]. The generation of nanoparticles in different solutions, particularly in pure water or deionised water, has received much attention from researchers in the field of nanoparticle generation because pure water is a suitable environment for the synthesis of nanoparticles and is free from any contamination.

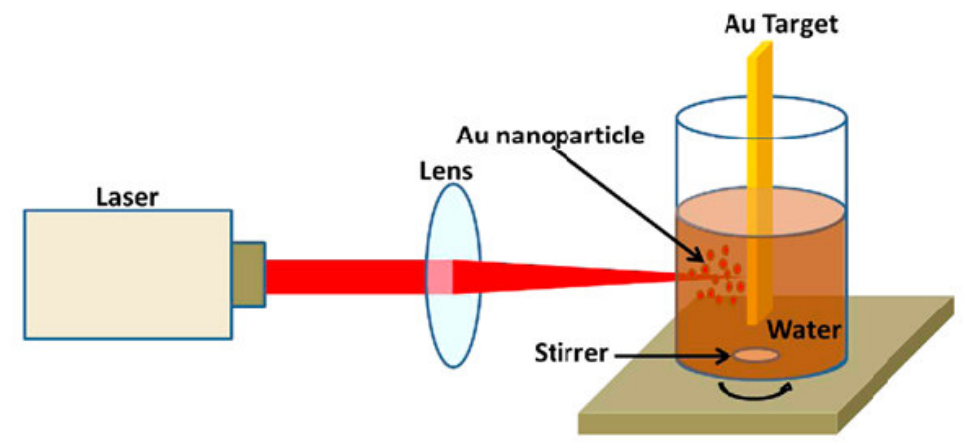

Schematic diagram of the experimental setup of PLAL

Laser ablation of solids in liquids is an effective technique with considerable potential in the generation of nanocrystals, which allows multilateral design through choosing appropriate solid target materials and confining liquids [11]. The response of different liquid solutions in the generation of nanoparticles varies considerably.

In this work, gold colloids were prepared by pulsed laser ablation of high purity gold plate immersed in deionized water and $10 \mu \mathrm{M} \mathrm{NaCl}$ solution. We study the change in the size distribution of nanoparticles after the ablation process for a month. Size distribution of obtained nanoparticles was measured using dynamic light scattering (DLS) method. TEM images of nanoparticles were obtained at at different time intervals after laser ablation process. 


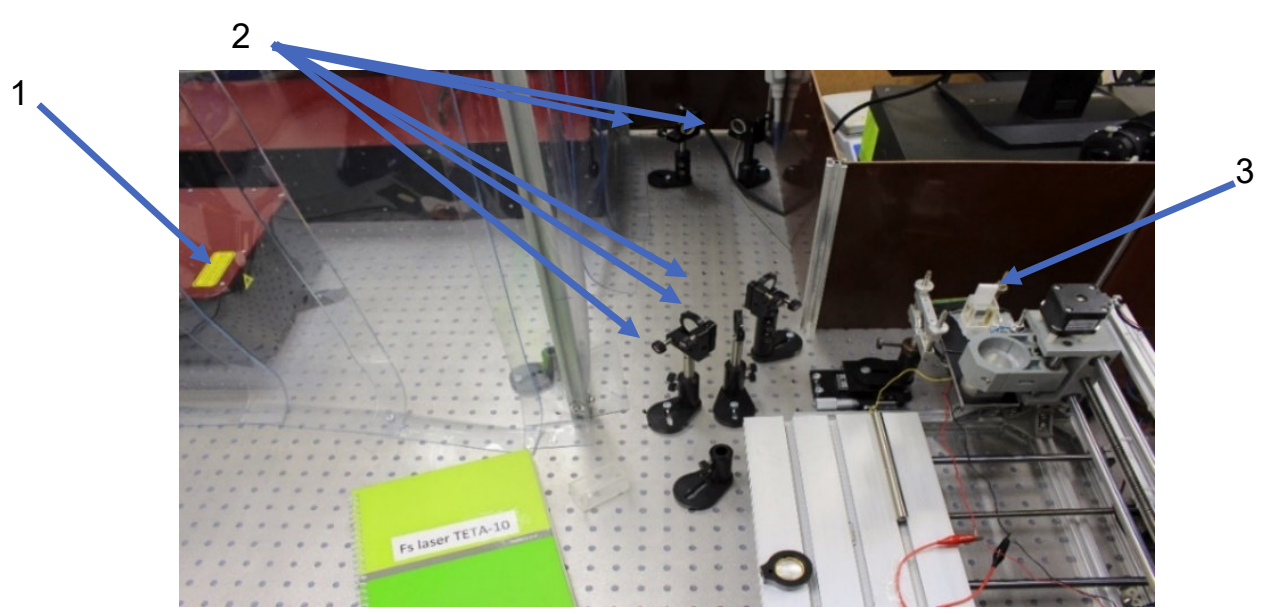

Experimental setup

1. Fs laser (270 fs, $1030 \mathrm{~nm}, 1-100 \mathrm{kHz})$.

2. Mirrors.

3. A vessel with the target.

At the first stage, aqueous and $10 \mu \mathrm{m} \mathrm{NaCl}$ solution were synthesized of au nanoparticles by ablation of a solid target using the teta-10-compals femtosecond laser system.

Next, a systematic study was conducted of the dependence of their dimensional, morphological properties on the parameters of ablating laser radiation: energy, duration of the synthesis process; as well as the type of liquid in which the synthesis is carried out (water, nacl solution) and the time after synthesis in the range from 5 minutes to 30 days using a combination of methods, such as scanning transmission electron microscopy (size, morphology), dls (dynamic light scattering).

\section{RESULTS}

In this communication, we study the change in the size distribution of nanoparticles after the ablation process over time. From the end of ablation to the $21^{\text {st }}$ day. Size distribution of obtained nanoparticles was measured using dynamic light scattering (DLS) method. TEM images of nanoparticles were obtained at regular intervals after laser ablation process.

Table 1 Parameters of laser radiation

\begin{tabular}{|l|l|l|l|l|l|}
\hline \multicolumn{1}{|c|}{ Liquid } & Energy,J & Laser WL,nm & Power,W & RR, Hz & Total Duration,mins \\
\hline $\begin{array}{l}\text { Deionised water; } \\
10 \mu \mathrm{M} \mathrm{NaCl}\end{array}$ & $100 \mu$ & 1030 & $1000 \mathrm{~m}$ & $10 \mathrm{k}$ & $40 \mathrm{~min} ;$ \\
\hline
\end{tabular}

It should be noted that in laser ablation mechanism selection of parameters of laser radiation (wavelength, pulse duration, its power) allows you to finely control the process of formation of nanostructures, and therefore, allows you to obtain materials with the required characteristics [11].

STEM images of Au nanoparticles in deionised water produced by PLAL at different time intervals after ablation (a) 1 hour, (b) 3 days are illustrated at Figure 1.

Figure 2 illustrates size distribution of Au nanoparticles in deionized water after ablation at different time intervals. 

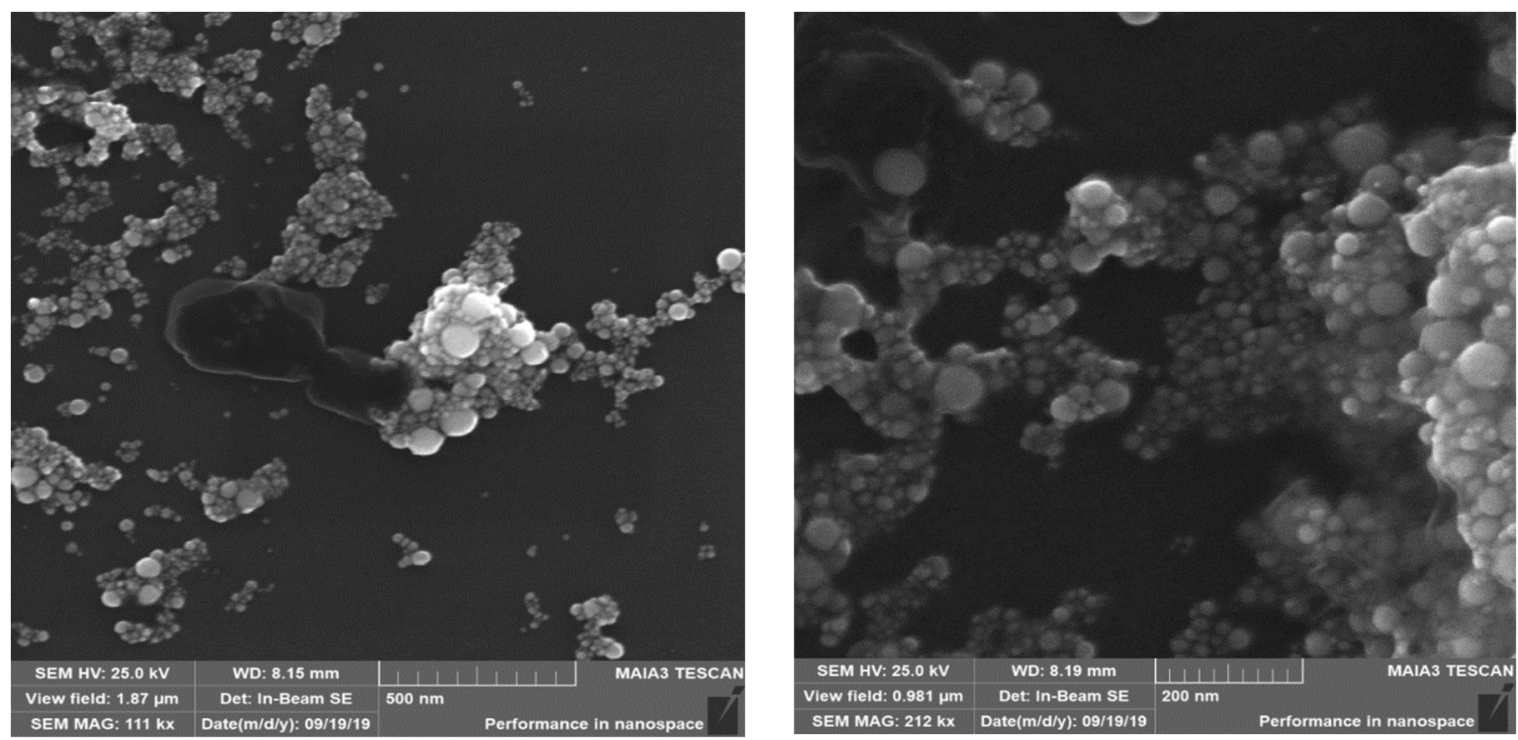

Figure 1 STEM images of Au nanoparticles in deionised water produced by PLAL at different time intevals after ablation (a) 1 hour, (b) 3 days

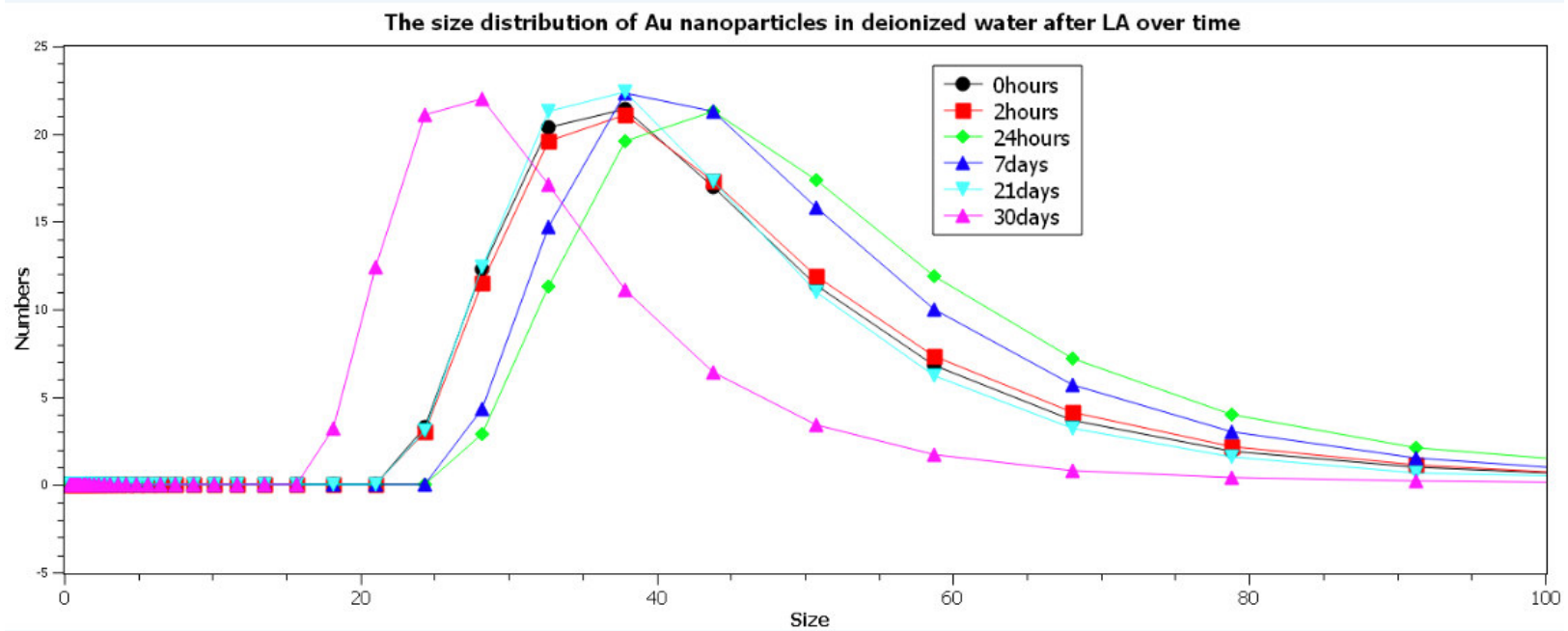

Figure 2 Size distribution of Au nanoparticles in deionized water after ablation at different time intervals

The size distribution of Au nanoparticles in $10 \mu \mathrm{M} \mathrm{NaCl}$ solution after LA over time

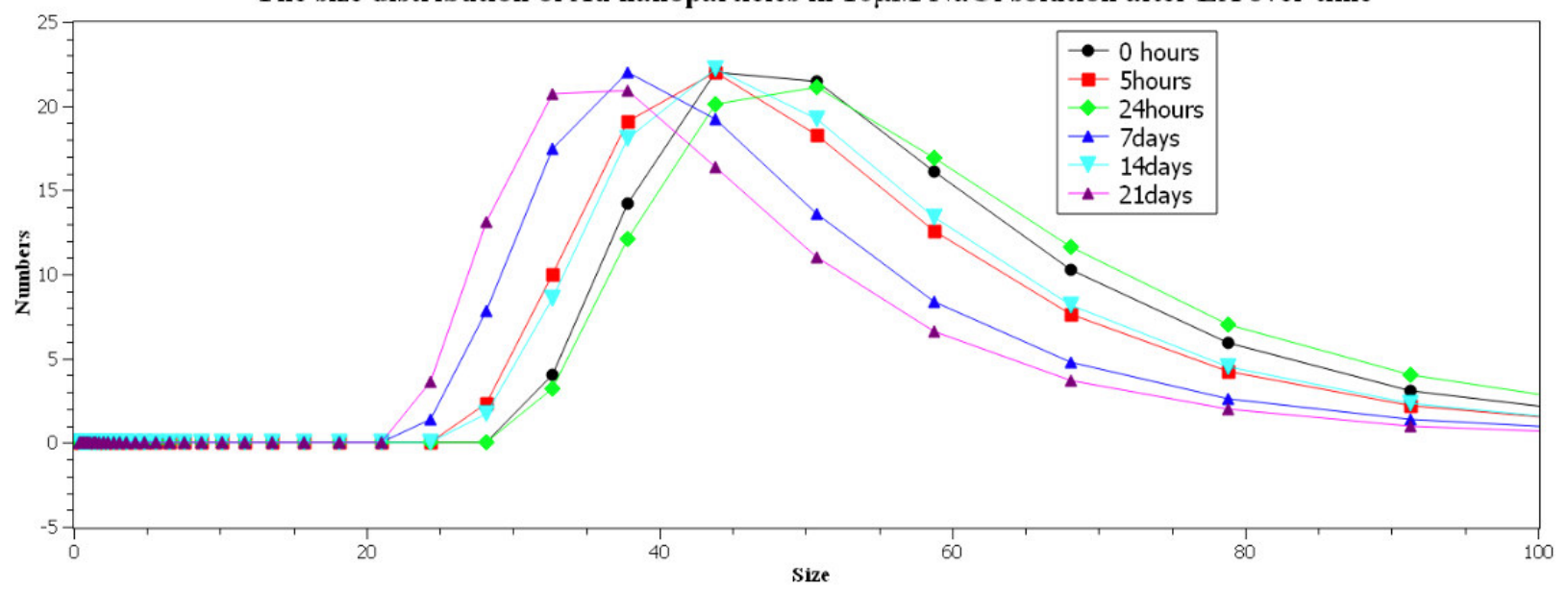

Figure 3 Size distribution of Au nanoparticles in $10 \mu \mathrm{M} \mathrm{NaCl}$ solution after ablation at different time intervals 
Figure 3 shows size distribution of Au nanoparticles in $10 \mu \mathrm{M} \mathrm{NaCl}$ solution after ablation at different time intervals.

\section{CONCLUSION}

Colloidal solutions of plasmonic nanoparticles, such as gold and titanium nitride, have a number of unique properties, in particular, resonant absorption and scattering of light, as well as a huge amplification of the electromagnetic field near the surface, which makes them very effective both in catalysis and in biomedical applications. However, arbitrary transformations of the size, morphology and structure of nanoparticles during synthesis, and most importantly after its completion, during storage, temperature fluctuations and dilution of solutions is a significant problem. Uncontrolled and often unpredictable transformations lead to a loss of useful properties and a decrease in the reproducibility of the properties of nanoparticles, and hinder their practical application in biomedicine.

From this work, we can conclude that in the long-term time range, colloidal solutions of gold nanoparticles in water and $\mathrm{NaCl}$ solution remain stable. Sizes of nanoparticles range from 20 to $60 \mathrm{~nm}$.

\section{ACKNOWLEDGEMENTS}

This work was financially supported from russian science foundation (grant № 19-14-00171).

\section{REFERENCES}

[1] JAIN, P. K., LEE, K. S., EL-SAYED, I. H. \& EL-SAYED, M. A. Calculated absorption and scattering properties of gold nanoparticles of different size, shape, and composition: applications in biological imaging and biomedicine. J. Phys. Chem. B 110, 7238-7248 (2006).

[2] NIE, S. Probing single molecules and single nanoparticles by surface-enhanced Raman scattering. Science (80-. ). 275, 1102-1106 (1997).

[3] CUI, C., GAN, L., HEGGEN, M., RUDI, S. \& STRASSER, P. Compositional segregation in shaped Pt alloy nanoparticles and their structural behaviour during electrocatalysis. Nat. Mater. 12, 765-771 (2013).

[4] DREADEN, E. C., ALKILANY, A. M., HUANG, X., MURPHY, C. J. \& EL-SAYED, M. A. The golden age: gold nanoparticles for biomedicine. Chem. Soc. Rev. 41, 2740-2779 (2012).

[5] OldENBURG, S., AVERITT, R., WESTCOTT, S. \& HALAS, N. Nanoengineering of optical resonances. Chem. Phys. Lett. 288, 243-247 (1998).

[6] BALASUBRAMANIAN, S. K. et al. Characterization, purification, and stability of gold nanoparticles. Biomaterials 31, 9023-9030 (2010).

[7] FOJTIC, A. \& HENGLEIN, A. LASER Ablation of Films and Suspended Particles in a Solvent: formation of Cluster and Colloid Solutions. Berichte der Bunsengesellschaft für Phys. Chemie 97, 252 (1993).

[8] SYLVESTRE, J. et al. Surface Chemistry of Gold Nanoparticles Produced by Laser Ablation in Aqueous Media. J. Phys. Chem. B 108, 16864-16869 (2004).

[9] PATIL, P., D. PHASE, S. KULKARNI, S. GHAISAS, S. KULKARNI, S. KANETKAR, S. OGALE and V. BHIDE, Physical Review Letters, 1987. 58(3): p. 238.

[10] MUSAEV, O., M. DRIVER, E. SUTTER, A. CARUSO, J. WROBEL and M. KRUGER, Applied Physics A, 2013. 113(2): p. 355-359.

[11] WAFAA MUSTAFA ALY SOLIMAN, Fundamental Studies on the Synthesis Dynamics of Nanoparticles by Laser Ablation in Pressurized Water, a dissertation submitted in partial fulfillment of the requirements for the degree of doctor of engineering, department of electrical engineering and computer science, Nagoya Univeristy, Nagoya, Japan, March 2011. 